\title{
Ab Initio Insights into the Interaction Mechanisms between Boron, Nitrogen and Oxygen Doped Diamond Surfaces and Water Molecules
}

Carlos Ayestarán Latorre, ${ }^{1,2,}{ }^{*}$ James P. Ewen, ${ }^{1}$ Daniele Dini ${ }^{1}$, M. C. Righi ${ }^{3}$

1 - Department of Mechanical Engineering, Imperial College London, London, SW7 2AZ, United Kingdom

2 - Department of Materials, Imperial College London, London, SW7 2AZ, United Kingdom

3 - Department of Physics and Astronomy, University of Bologna, 40127 - Bologna, Italy

*- Corresponding author email: carlos.ayestaran-latorre17@imperial.ac.uk

\section{ABSTRACT}

Diamond and diamond-like carbon coatings are used in many applications ranging from biomedicine to tribology. A wide range of dopants have been tested to modify the hydrophilicity of these surfaces, since this is central to their biocompatibility and tribological performance in aqueous environments. Despite the large number of experimental investigations, an atomistic understanding of the effects of different dopants on carbon film hydrophilicity is still lacking. In this study, we employ ab initio calculations to elucidate the effects of $\mathrm{B}, \mathrm{N}$, and $\mathrm{O}$ dopants in several mechanisms that could modify interactions with water molecules and thus hydrophilicity. These include the adsorption of intact water molecules on the surfaces, minimum energy pathways for water dissociation, and subsequent interactions of hydrogenated and hydroxylated surfaces with water molecules. We find that all of the dopants considered enhance hydrophilicity, but they do so through different means. Most notably, $B$ dopants can spontaneously chemisorb intact water molecules and increase its interactions in $\mathrm{H}$-bond networks. 


\section{KEYWORDS}

Hydrophilicity, diamond, DFT calculations, boron, nitrogen, oxygen.

\section{INTRODUCTION}

Carbon-based coatings, such as diamond-like carbon (DLC) are widely researched in fields ranging from biomedicine [1,2] to tribology [3,4], due to their high biocompatibility, ultra-hardness, low-friction, and high wear resistance capabilities. They are now utilised in numerous industrial applications such as artificial joints [1,2] and engine components $[3,4]$. In aqueous or humid air environments, the interaction of DLC surfaces with water molecules may modify or even determine its tribological properties [5]. At low relative humidity, hydrophobic DLC films protected by a selfassembled monolayer showed lower adhesion and friction compared to hydrophilic DLC films [6]. One the other hand, hydrophilic DLC films generally show lower friction at high relative humidity [7]. Ab initio calculations have shown that this is because such surfaces favour the formation of a wetting layer that prevents the formation of covalent bonds across the opposing sliding surfaces and makes them slippery [8-10], thus reducing friction in humid conditions.

The wettability of DLC surfaces is a complex phenomenon, affected by macroscopic properties such as surface energy [11], as well as atomic-scale factors including polarity, dangling bonds, $\mathrm{sp}^{3} / \mathrm{sp}^{2}$ ratio [12] and surface hydrogenation [13]. Experimental studies of the wettability of DLC coatings show a wide range of values depending on the surface roughness [14], texture [15], and deposition methodology [16]. These competing factors has complicated the fundamental understanding of DLC wettability and has prevented the systematic development of coatings with improved performance. A popular approach to increasing the hydrophilicity of DLC is doping it with other elements in order to increase surface energy or polarity [17-20].

As well as increasing the wettability, the doping of DLC with non-metallic elements such as boron, nitrogen, oxygen, and silicon is performed to improve other properties [21], such as biocompatibility $[22,23]$ and electrical conductivity $[23,24]$. Several studies have been devoted to investigating the effects of dopants on friction and wear [3]. Large decreases in the friction coefficient of boron-doped DLC (0.04) compared to undoped DLC (0.22) have been reported in humid air [3]. More recent studies have 
shown that low concentrations of boron (2.6 at.\%) in DLC resulted in better friction performance (at the cost of slightly higher wear rates) than undoped DLC in ball-ondisk tribometer experiments at high relative humidity (32-56 \%) [25]. However, it has also been shown that the wear rate of micro- and nano-crystalline diamond increases linearly (by a factor of three) with boron concentration (between 0.6 and 2.8 at.\%) in ball-on-disk experiments in humid air [26], whereas friction was similar in all cases. Nanoscale atomic force microscopy (AFM) [27] and ball-on-disk [28] found no correlation between nitrogen dopant concentrations in DLC and the friction coefficient, but high concentrations deteriorated wear resistance. On the other hand, improved friction performance of DLC with a relatively low N/C ratio (0.08) has been reported compared to undoped DLC at high relative humidity (40-45\%) [29]. While oxygencontaining DLC films have been linked with increased wettability and wear rates [30], as well as increased hardness due to a raised ratio of aromatic to olefinic sp2 $\mathrm{C}=\mathrm{C}$ bonds [31] compared to nondoped DLC, their tribological performance has not been widely studied. However, silicon and oxygen co-doped DLC have shown excellent tribological properties $[32,33]$.

Several experimental studies have investigated the interactions of doped and undoped DLC coatings with water interfaces. DLC has a typical water contact angle (WCA) of $80^{\circ}$ [34-36], although hydrogen-free tetrahedral amorphous carbon (ta-C) can display a lower angle of $\sim 60^{\circ}$ [34]. Surfaces with WCA greater than $90^{\circ}$ are generally regarded as hydrophobic, while those with lower WCAs are hydrophilic [37]. Wettability of borondoped DLC has been shown to change nonmonotonically with dopant concentration [25]. An increase in the hydrophilicity (WCA increased from $60^{\circ}$ to $68^{\circ}$ ) was observed moving from undoped DLC to 2.6 at. \%, which was largely attributed to the formation of polar B-C bonds. At intermediate dopant concentrations (9.2 at.\%,), the WCA increased to $72^{\circ}$, attributed to the appearance of less polar B-H and C-H bonds. When the boron concentration was further increased (25.8 at.\%), a much higher hydrophilicity of the surface, with a WCA of $35^{\circ}$ [37], was associated with B-O bonds that could enhance hydrogen-bonding. Most experiments have shown that higher nitrogen concentration lead to increased hydrophilicity. A 5.3 at.\% nitrogen concentration reduced the WCA of DLC from $80^{\circ}$ to $74^{\circ}$ [38], while higher concentrations of nitrogen (12.7 at.\%) showed a lower WCA of $63^{\circ}$ [36]. However, 
reports are unclear whether this is mostly due to an increase in the polar [34] or the dispersive [38] component of surface energy. $O$ doping has been shown to increase DLC hydrophilicity, with low WCA $\left(63^{\circ}\right)$ having been reported at high oxygen concentrations (11 at.\%) [20]. Infrared spectroscopy indicated the presence of carbonyl oxygens, although there were no reports on the chemical state of the residual oxygens in the DLC after etching [20]. It is also noteworthy that extremely hydrophilic DLC films, with WCA as low as $3^{\circ}$, have been produced using piranha treatments [6]. This was attributed mainly to the formation of oxygen containing hydroxyl, carbonyl and epoxy groups on the surface [6].

Despite this large number of experimental studies, a molecular-scale understanding of the physicochemical mechanisms driving the increase in hydrophobicity by different dopants is still lacking. This could reconcile the differences between experimental results, as well as facilitate the design of improved coatings. Previous investigations have shown that the preference of water molecules to physisorb or chemisorb on different types of DLC coatings determined their friction coefficient in non-lubricated contacts as a function of water vapour pressure $[39,40]$. Thus, understanding the role of dopants on water adsorption mechanisms could further explain their effect on tribological properties.

Several ab initio studies by Righi and co-workers $[9,10,41,42]$ have revealed many molecular-scale details of the interactions between water and diamond surfaces, which are locally representative of DLC. A recent density functional theory (DFT) study by Kajita and Righi [43] found that Si doping of DLC lowered the energy barrier for water dissociative adsorption, which was proposed as a mechanism for increased wettability through the surface hydroxyls interacting with water molecules. Subsequent ab initio molecular dynamics (AIMD) simulations [8] studied this process in water molecules confined between sliding diamond surfaces. These AIMD simulations supported experimental observations that water molecules can form a thin layer between the passivated surfaces and act as a boundary lubricant [44]. The fundamental mechanism of silicon atoms acting as catalytic sites for hydroxylation was also consistent with experimental observations of superlow friction coefficient of silicon-doped DLC in humid environments [45]. 
The role of other non-metal dopants on the interactions between DLC and water have been less widely studied with ab initio methods. Previous AIMD simulations [46] suggested that the hydrophilic character of boron-doped diamond depends on the surface termination. Surfaces covered in carbonyl oxygens presented strong interaction with water molecules, while hydrogenated ones were markedly hydrophobic. Mixtures of hydroxyls and hydrogen on the surface, which could be formed from the dissociative adsorption of water molecules, recovered some of the hydrophilicity. However, these simulations for boron-containing systems were not compared to undoped DLC. Moreover, the dopants were embedded in inner layers and as such, their direct effect on water adsorption was not studied. Other ab initio studies have highlighted the local effects of dopants on chemical environment and adsorption energies for other molecules on diamond surfaces [47-49]. One of these studies suggested that the hydrophobicity of boron-doped DLC surfaces increases as the boron atom is closer to the surface [47]. However, this observation disagrees with the more recent experimental results discussed above [25].

In this study, we will use ab initio methods to investigate the nature and strength of the interaction between $\mathrm{B}-, \mathrm{N}-$, and O-doped diamond (BDD, NDD and ODD, respectively) surfaces and water molecules. The $\mathrm{B}, \mathrm{N}$, and $\mathrm{O}$ dopants are located at the most energetically stable, exposed substitutional sites, which were identified by screening several possibilities in the outer layers. A similar protocol to that recently employed by Kajita and Righi for Si dopants [43] will be followed. We will employ DFT to investigate the effects of the different dopants, located in the outer layers, on the molecular and dissociative adsorption of water molecules, dissociative adsorption, and the role of dissociated hydrogens and hydroxyls on hydrogen bonding with further water molecules. As we focus on substitutional sites, the $O$ will be located in an epoxy configuration, as opposed to the more often studied carboxylic locations; similar epoxy configurations have been previously studied [50]. Through these calculations, we uncover the distinct roles of the different dopants in altering the wettability of carbonbased films.

\section{METHODOLOGY}

We employ a supercell consisting of a 10-layer thick (001) slab with a $4 \times 3$ in-plane size (120 atoms) and a vacuum region of $\sim 14 \AA$ between each periodic replica in the 
[001] direction. The surfaces of the slab present a $2 \times 1$ dimer reconstruction [51] that results in alternating rows and trenches of $\mathrm{sp}^{2}$-and $\mathrm{sp}^{3}$-bonded carbon atoms. This surface has frequently been used in ab initio studies as a simple model for hydrogenfree DLC $[43,46]$. It is representative of the $\mathrm{sp}^{2}$ layers often present at the surface of DLC coatings [52].

In each supercell, one dopant atom was located at a substitutional lattice site in the outer layers. While $B[53]$ and $N[54,55]$ atoms are known from previous studies to favour substitutional sites, it is not clear whether this is also the case for $\mathrm{O}$ atoms. IR absorption spectra of O-containing DLC films often indicate the presence of carbonyl oxygens [20] $(C=O)$, but other studies found $O$ incorporation in bridge positions (two $\mathrm{C}-\mathrm{O}$ bonds) to be more stable [46], or have suggested the presence of epoxy groups $[6,50]$. We tested the stability of $O$ in substitutional sites, to maintain consistency with the other dopants. The preferred position for all dopants, as detailed below, is in the surface heterodimer. One dopant atom is added to each system, which corresponds to a dopant concentration in the outer layer of 8.3 at.\%. This concentration falls within the ranges employed in the experiments discussed in the Introduction.

DFT calculations are carried out using the plane-wave software QUANTUM ESPRESSO [56]. Quantum ESPRESSO has been used extensively to study a wide range of material properties and processes [57]. We employ the generalized gradient approximation developed by Perdew, Burke and Ernzerhof (PBE) [58] for the exchange-correlation functional in structural relaxations. After testing, we found that it better represented the bulk properties of diamond compared to the local-density approximation (LDA) [59] and Perdew-Wang [60] (PW91) functional. Nonetheless, van der Waals $(\mathrm{vdW})$ contributions are expected to play a significant role in moleculesurface interactions. To account for them, the most stable PBE-relaxed geometries were subsequently further relaxed with DFT-D2[61,62] and DFT-D3[63] dispersion corrections, as detailed throughout the text. Although both corrections are expected to yield similar values, the inclusion of both DFT-D2 and DFT-D3 results could serve as an extra benchmark given the semiempirical, non-electronic nature of the corrections and sparse implementation in similar systems. All of the calculations include spin polarization. The electronic wave functions are expanded on a plane-wave basis set with a cutoff energy of $30 \mathrm{Ry}$, and the ionic species are described by ultra-soft 
pseudopotentials [64]. The $k$ points in the Brillouin zone are sampled by means of a 2 $\times 3 \times 1$ Monkhorst-Pack grid [65]. A Gaussian smearing with a 0.01 Ry spreading value was applied for Brillouin-zone integration. The convergence threshold for geometry optimization was $10^{-4}$ Ry for the total energy and $10^{-3}$ Ry/Bohr per atom for ionic forces. Visualisations were made using the OVITO software [66].

The adsorption energy of a water molecule on the surface was calculated as $E_{a d s}=$ $E_{\text {tot }}-E_{\text {surf }}-E_{\mathrm{H}_{2} \mathrm{O}}$, where $E_{\text {tot }}, E_{\text {surf }}$ and $E_{\mathrm{H}_{2} \mathrm{O}}$ are the energies of the adsorbate system, surface slab and water molecule, respectively. $E_{\text {tot }}$ and $E_{\text {surf }}$ are calculated using the above described supercell, while the water molecule energy was calculated using a cubic cell with a length of $12 \AA$. A recent study of fluorine-terminated diamond surfaces has shown that the contact angle of a water droplet (from classical MD simulations) is inversely correlated with the adsorption energy of a single water molecule (from DFT calculations) [67]. Thus, we expect the calculated adsorption energies to give a direct indication as to the wettability at larger scales.

All computational parameters were optimised in convergence tests. The converged diamond lattice parameter with our setup (obtained by fitting the Birch-Murnaghan [68] equation of state) is $3.577 \AA$. This is in close agreement with the experimental value of $3.567 \AA$ [69] and with a similar accuracy to that provided by more expensive methods such as hybrid functionals [70]. The diamond lattice was used to build the slab as described above, which was then relaxed; the positions of the atoms in the bottom layer were then fixed to resemble the bulk for all subsequent calculations. The final setup yields converged values for $E_{\text {ads }}$ that are within $\sim 0.01 \mathrm{eV}$ of our most accurate benchmarks, and a surface energy of $4.67 \mathrm{~J} / \mathrm{m}^{2}$, which agrees closely with values previously reported in the literature $[71,72]$.

Reaction paths for the transition between water adsorption and dissociative chemisorption were obtained with the nudged elastic band (NEB) method within the climbing image scheme to determine the transition state $[73,74]$ with PBE calculations. The NEB method determines the intermediate steps for the transition between two stable states corresponding to the minimum energy path (MEP), and correspondingly determines the energy barrier of such transition. 8 images were employed for each 
reaction path, optimized with a broyden scheme and a string constant of 0.7 in Hartree atomic units. The reaction path was first converged without the climbing image scheme, and then the method was implemented to obtain accurate energy barriers.

\section{RESULTS}

\subsection{Preferred incorporation sites for dopants}

First, we identify the most favourable locations for the different dopants by comparing the stability of different substitutional sites in the exposed first (a), second (b) and third (c) outer layers, as shown in Fig. 1.

a)

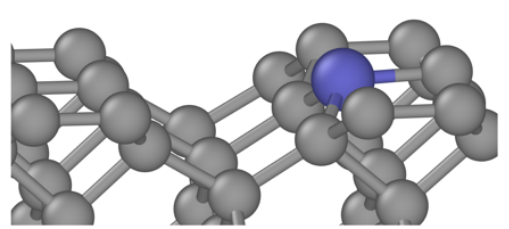

B: $\quad 0.0 \mathrm{eV}$ b)

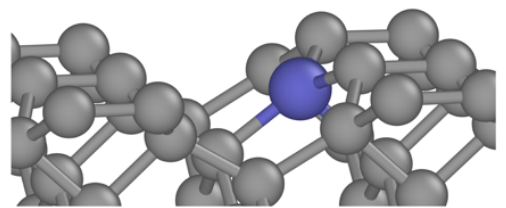

$+0.40 \mathrm{eV}$

$+2.30 \mathrm{eV}$

$+4.24 \mathrm{eV}$ c)

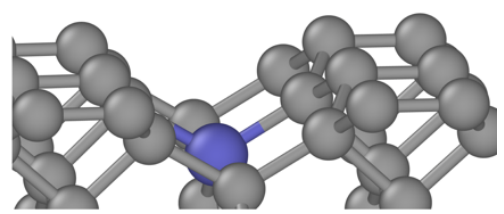

$+0.68 \mathrm{eV}$

$+2.87 \mathrm{eV}$

$+0.44 \mathrm{eV}$

Figure 1. Energy differences (PBE) are relative to the most stable configuration, which happens to be in the top layer dimers (a) for all dopants considered.

All dopants preferred to incorporate in place of a $\mathrm{C}$ in a surface heterodimer. This can be rationalised in terms of valence electrons and Löwdin partial charges [75]. Although we do not expect the absolute Löwdin charges to be accurate, the differences should be useful for interpreting physical trends. When the $B$ dopant is added, the $\operatorname{dimer} C=C$ double bond turns into a C-B single bond, such that it maintains an even number of electrons (six) in its valence. This was observed in a relaxation of the dimer bond length (from $1.38 \AA$ to $1.57 \AA$ ) and in the fact that, in this (a) location, the $B$ atom presented was positively charged compared to the tetragonal, $\mathrm{sp}^{3}$-bonded (b) and (c) sites in the second and third layers; approximately $+0.3 \mathrm{e}$ in (a) compared to $0.0 \mathrm{e}$ in (b) and (c)). A similar reasoning applies to the $\mathrm{N}$ atom, which can complete its outer shell by forming three bonds in the (a) location, instead of having to donate electrons to form four bonds in the second and third layer positions. A look at its corresponding Löwdin charges shows indeed a more negative partial charge if $-0.2 \mathrm{e}$ in (a) compared to $0.0 \mathrm{e}$ in the (b) and (c) positions. The preference for $\mathrm{sp}^{2}$ bonding of $\mathrm{N}$ dopants has also been reported experimentally [76]. The $\mathrm{O}$ dopant has a different effect, as it only 
forms two bonds when relaxed in each of the three positions. The resulting disruption in the slab due to the bond breaking is minimized in the (a) site, in which it breaks the dimer and adopts a bridge position between $\mathrm{C}$ atoms in the second layer, similar to that observed in Ref. [46], and forms epoxy groups as suggested in Ref. [6]. The relaxed coordinates for all dopants are reported in Fig. 2. It was found that $B$ and $N$ barely disrupt the surface geometry, which can be understood, at least to a first approximation, by considering that they have similar covalent radii as carbon [77] (within $\sim 20 \%$ ). By contrast, Si atoms, which were found to yield sizeable deformations to the same surface [43], have a covalent radius that is $\sim 60 \%$ larger than for carbon.

In their relaxed configurations, the above stated Löwdin charges suggest the $\mathrm{O}$ and $\mathrm{N}$ dopants are negatively charged, while the $\mathrm{B}$ dopant is positively charged. This agrees with their Pauling electronegativities [69]: $\mathrm{O}(3.44)>\mathrm{N}(3.04)>\mathrm{C}(2.55)>\mathrm{H}(2.20)>\mathrm{B}$ (2.04), as the oxygen and nitrogen are more electronegative than surrounding carbon atoms, while the opposite is true for boron.
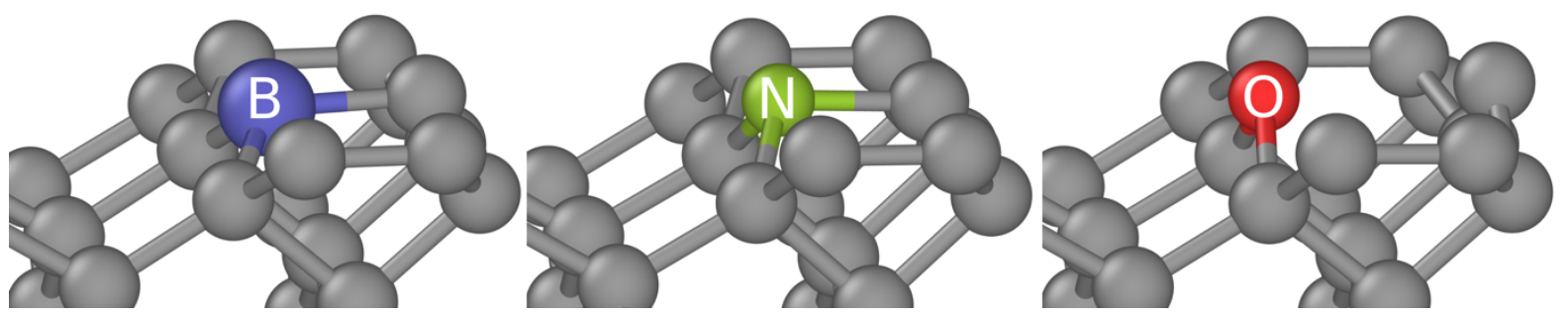

Figure 2. Relaxed positions of $\mathrm{B}, \mathrm{N}$ and $\mathrm{O}$ dopants (in blue, green and red, respectively) incorporated in the surface dimers. The oxygen can be seen to break the surface dimer.

\subsection{Effects of dopants on water adsorption}

Water molecules were then directly adsorbed on the doped and undoped diamond slabs by placing them on various probe positions and orientations to find the most favourable configurations. The most stable conformations and corresponding adsorption energies are shown in Fig. 3. 


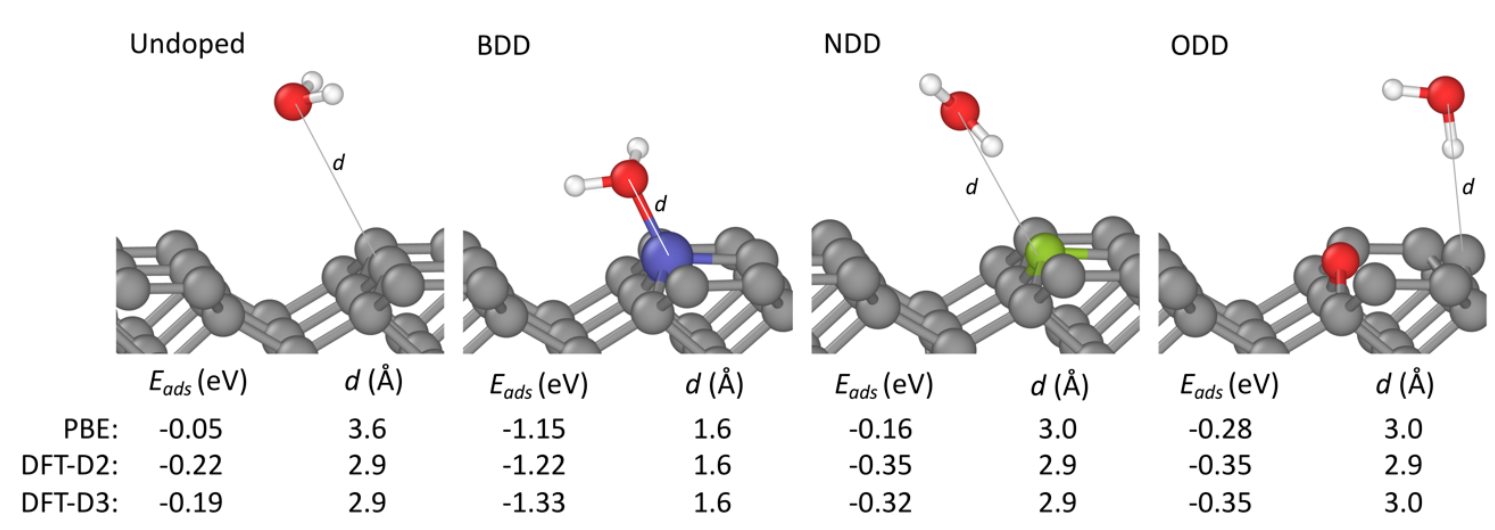

Figure 3. Relaxed water adsorption geometries with corresponding adsorption energies (for PBE and vdW corrections DFT-D2 and DFT-D3), $E_{a d s}$, and distances to surface, $d$, as indicated in the images. Note that previous a previous DFT study has shown that $E_{\text {ads }}=-0.12 \mathrm{eV}$ for Si-doped DLC [43]. $\mathrm{B}$ is shown in blue, $\mathrm{N}$ in green, $\mathrm{O}$ in red, $\mathrm{H}$ in white and $\mathrm{C}$ in grey.

The inclusion of $\mathrm{vdW}$ corrections does significantly increase the molecule-surface interactions. It should be observed, however, that the overall sorting of the energies remains largely the same. Similarly, the overall geometries and distances to the surface remain mostly unchanged, with the exception of the adsorption of a water molecule on the bare DLC surface; in this case, the vdW term pulls the molecule slightly closer to the surface above the trench between dimer rows.

In agreement with previous DFT results [43], water shows only a weak interaction with the undoped surface (DFT-D3: $-0.19 \mathrm{eV}$ ). The water molecules interact more strongly with a (negatively partial charged) $\mathrm{N}$ dopant (DFT-D3: $-0.32 \mathrm{eV}$ ) by pointing one of its positively partial charged $\mathrm{H}$ atoms towards it. This observation agrees with experimental results that link increased hydrophilicity to the presence of polar C-N bonds [38] (and $\mathrm{N}-\mathrm{H}$ bonds, as discussed below). It is perhaps surprising that this conformation is not reproduced with an $\mathrm{O}$ dopant. The interaction strength is of similar magnitude (DFT-D3: $-0.35 \mathrm{eV}$ ) compared to $\mathrm{N}$, despite $\mathrm{O}$ having slightly higher electronegativity. This is because one of the water $\mathrm{H}$ atoms is pulled towards the surface $O$ atom, while the other is pulled towards the $C$ in the bridge position that results from the broken dimer. The preferential interaction of the water molecule with the less negatively charged $C$ atom suggests a more dispersive rather than polar nature [38]. Previous ab initio [46] studies have remarked on the role of surface 
carbonyl $\mathrm{O}$ atoms on enhancing hydrophilicity via hydrogen bonding. Fig. 3 suggests that this could also be the case for epoxy $\mathrm{O}$ atoms although the conformation is somewhat different.

An altogether different behaviour is observed in the B dopant case. As opposed to the physisorption that occurs in the other systems, the water interacts with the $B$ dopant through strong chemisorption (DFT-D3: $-1.33 \mathrm{eV}$ ). The energy gain from filling the boron outer shell drives the water $\mathrm{O}$ to form a dative O-B bond. Löwdin analysis shows that the bond formation results in a less negatively charged $\mathrm{O}(-0.4 \mathrm{e})$ compared to in an isolated water molecule (-0.7 e). Previous DFT studies had similarly shown [47] that there is a preference of $B$ dopants for tetrahedral coordination in the outer layer. However, previous ab initio studies using hydrogenated BDD surfaces [47] have shown weaker adsorption energies (DFT-D3: $-0.58 \mathrm{eV}$ ) for tetrahedral $\mathrm{B}$ atoms in the outer layer. This highlights the importance of surface hydrogenation on the wettability of doped DLCs.

The strong chemisorption observed for BDD suggests that a different mechanism may govern the wettability enhancement compared to other dopants. While other dopants, like $\mathrm{N}, \mathrm{O}$, and $\mathrm{Si}\left(E_{a d s}=-0.12 \mathrm{eV}\right)$ [43], may modify water-surface physisorption and reaction barriers towards surface hydroxylation, $B$ directly captures a water molecule in a process with no energy barrier and a high energy benefit. This water molecule could potentially participate in hydrogen bonding water molecules not directly bonded to the surface and enhance hydrophilicity [43]. This possibility will be further discussed in the following sections.

\subsection{Effects of dopants on water dissociative adsorption}

To study the effect of dopants on water dissociative adsorption mechanisms and stability, we adsorbed hydrogen and hydroxyl fragments on neighbouring surface atoms. Surface hydroxylation is known to attract further water molecules and increase hydrophilicity $[6,78]$.

A previous DFT (PBE) study for Si-doped DLC identified six stable configurations of the dissociated water molecule (Fig. 4 a1-a6). For the $\mathrm{N}$ and $\mathrm{O}$ dopants, many of these 
configurations were found to be unstable, whereas alternative configurations (a7-a10) where more stable. All of the analysed configurations, with a similar PBE setup to the mentioned study, are shown in Fig. 4 and the corresponding energies are shown in Table 1.
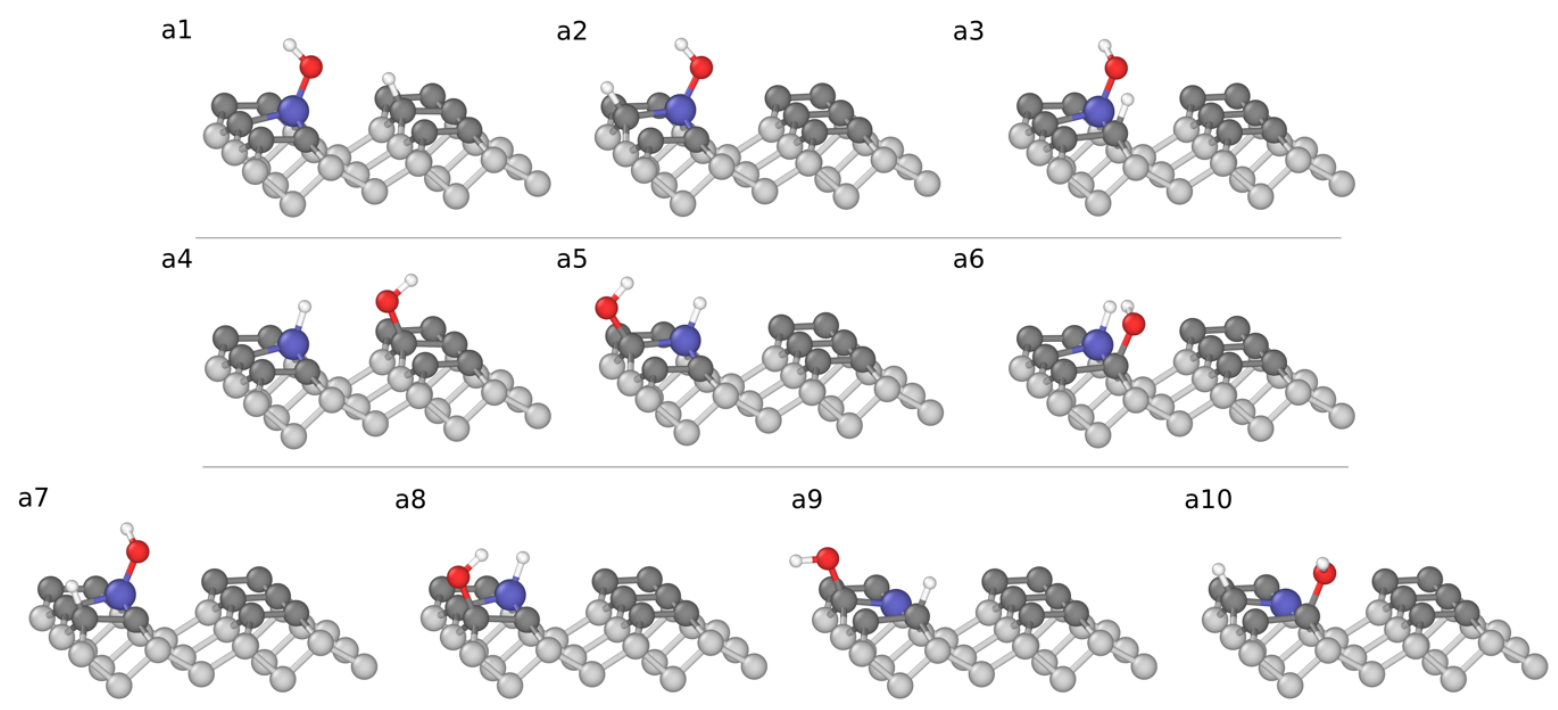

Figure 4. Water dissociative adsorption configurations considered in this study. In the image, relaxed geometries for the BDD slab. Equivalent geometries were relaxed for undoped, NDD and ODD surfaces, although not all were stable for the latter two. All corresponding energies are reported in Table 1. Geometries a7-10, with fragments placed diagonally across adjacent dimers, were not calculated for the silicon dopants by Kajita and Righi [43].

\begin{tabular}{lrrrrr} 
& BDD & NDD & ODD & DLC & SiDD [43] \\
\cline { 2 - 6 } a1 & -2.57 & - & - & -2.17 & -3.74 \\
a2 & -2.94 & - & - & -2.81 & -4.51 \\
a3 & -2.52 & - & - & -2.17 & -3.63 \\
a4 & -1.20 & -0.82 & - & -2.17 & -2.10 \\
a5 & -1.61 & -0.76 & -0.02 & -2.81 & -2.63 \\
a6 & -1.36 & -0.66 & - & -2.17 & -2.14 \\
a7 & -2.49 & - & - & -2.13 & \\
a8 & -1.24 & -0.87 & - & -2.13 & \\
a9 & -2.57 & -2.93 & -3.00 & -2.13 & \\
a10 & -2.84 & -2.79 & -2.71 & -2.13 &
\end{tabular}


Table 1. PBE dissociation energies (in eV) corresponding to configurations in Fig 4. All energies are with respect to the energy of isolated slab and water molecule systems. Configurations with a hyphen are not stable. Calculated energies for Sidoped diamond from Ref. [43] included for comparison.

The most stable configurations for each surface were further studied with vdW corrections. The corresponding results are reported in Table 2.

\begin{tabular}{lccr} 
& \multicolumn{3}{c}{ Adsorption energy (eV) } \\
& PBE & DFT-D2 & DFT-D3 \\
Undoped (a2) & -2.81 & -3.09 & -2.99 \\
BDD (a2) & -2.94 & -3.19 & -3.08 \\
NDD (a9) & -2.93 & -3.20 & -3.11 \\
ODD (a9) & -3.00 & -3.21 & -3.12
\end{tabular}

Table 2. Comparison of adsorption energies for the most stable dissociated configurations obtained without (PBE) and with (DFT-D2, DFT-D3) the two vdW corrections considered.

All of the studied configurations are stable in the boron case, with a2 being the most favourable. $\mathrm{N}$ and particularly $\mathrm{O}$ dopants prevent some of the dissociated configurations from being stable. No configurations that included $\mathrm{N}-\mathrm{OH}$ bonds were stable and the formation of additional bonds for oxygen was unfavourable due to its saturated outer shell. Generally, the doped surfaces from the present study yield only slightly more favourable energy gains compared to the clean diamond surface, by $\sim 0.1$ $\mathrm{eV}$ for both vdW-corrected calculations. This is in stark contrast to Si dopants, which resulted in a significantly more stable configuration when bonded with a hydroxyl ($4.51 \mathrm{eV}$ ) [43]. Some general tendencies can be observed and analysed.

Boron favours configurations where it is directly bonding with the hydroxyl group. Its most stable associated geometry (a2) results from saturating the resulting dangling bond in the heterodimer $\mathrm{C}$ with the $\mathrm{H}$ fragment, with a similar energy (DFT-D3: -3.08 $\mathrm{eV}$ ) as in the equivalent undoped case (DFT-D3: -2.99 eV). Hydrogenation of B dopant (a4-6) is energetically favourable, but is more than $1 \mathrm{eV}$ less favourable compared to hydroxylation. 
As for nitrogen, its complete valence shell causes the hydroxyls to detach when relaxed on top of it. It can adsorb hydrogens, on the other hand, by breaking the heterodimer bond, albeit with significantly less favourable energies than in the equivalent undoped geometries. The two most stable N-doped configurations, (a9-10), do not present any fragment adsorbed on the dopant itself, yet show a more favourable energy (PBE: $-0.8 \mathrm{eV}$ ) compared to their equivalent undoped geometries.

The oxygen in the bridge position remains non-reactive and does not bond with either of the fragments. Instead, it promotes water adsorption through the dangling bonds it introduces in its neighbours, favouring the geometry a9 and, to a lesser extent, a10. A similar effect happens for the $B$ and $N$ cases, which show high adsorption energies in the (a9) and (a10) configurations. This set of configurations is approximately $1 \mathrm{eV}$ more stable than their undoped counterparts.

None of the dopants studied in this contribution yield an energy gain as favourable as previously observed for $\mathrm{Si}$, which show differences of almost $2 \mathrm{eV}$. The large radius of Si complicates the $\pi$ overlapping required for the double bond in the heterodimer, which is then disrupted, and hence favours $\mathrm{sp}^{3}$ bonding to a hydroxyl; this in turn results in a saturated, highly stable configuration (a2). That mechanism is missing with the $\mathrm{N}$ and $\mathrm{O}$ dopants, which instead favour adsorption through the dangling bonds on the disrupted surface reconstruction. In the case of $\mathrm{B}$, although direct $\mathrm{OH}$ bonding (a2) is energetically favourable, the covalent radius is similar to $C$, so there is less disruption and less subsequent stabilisation by adsorption.

\subsection{Reaction path for water dissociation}

We also obtained the corresponding energy barriers for dissociation by finding the MEP using the NEB method [73,74], in this case only for PBE calculations. We consider as initial states of the reaction the stable water adsorption configurations shown in Fig. 3. The final states are those most stable for each system and dopant, namely a2 for the clean and BDD surfaces and a9 for the NDD and ODD cases. The results are summarised in Fig 5. 


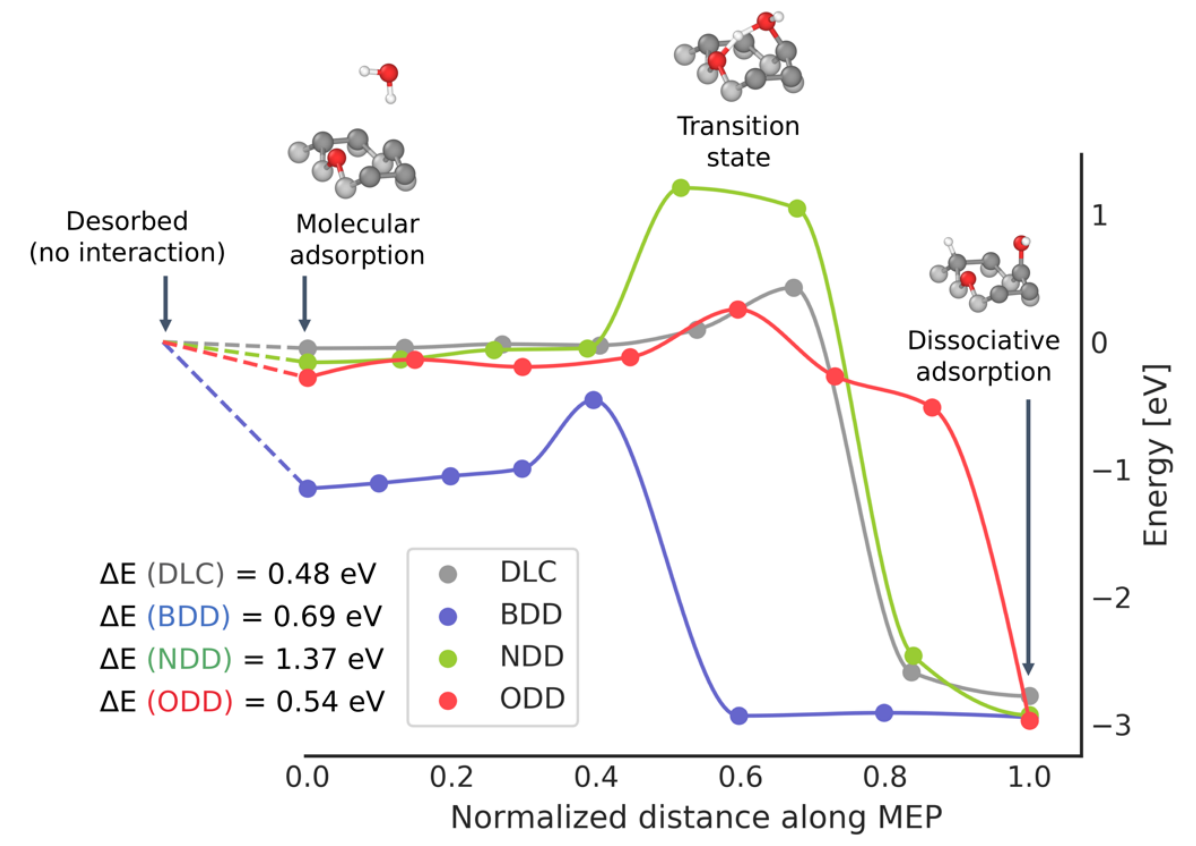

Figure 5. Reaction paths and energy barriers $\Delta E$ for water dissociation according to NEB calculations (PBE). The values of $\triangle E$ are reported relative to the corresponding initial adsorbed states. In contrast, the energies of the MEPs are plotted as differences to the desorbed configurations, to visualize the effect of adsorption energies (see Fig. 3 ) on the whole process from capturing a water molecule to a dissociated state. Inset images show the reaction path for water dissociation on ODD.

Undoped DLC showed the lowest barrier for dissociation $(0.48 \mathrm{eV})$, whereas ODD $(0.54 \mathrm{eV})$ and BDD $(0.69 \mathrm{eV})$ showed a slightly increased barrier height. In both of these cases, however, when also considering the energy gain for water molecular adsorption, the transition state is at a lower relative energy than on clean DLC. NDD, on the other hand, cause a large increase in the dissociation barrier $(1.37 \mathrm{eV})$ despite ending in the same configuration (a9) as the $O$ dopant case. We explain this behaviour by inspecting the transition state in the oxygen dopant case. We find that the hydrogen is pulled apart from the water molecule by the dopant, which results in a $\mathrm{O}-\mathrm{H} \cdots \mathrm{O} \mathrm{H}$ bonded transition state, with the $\mathrm{H}$ atom equidistant from both $\mathrm{O}$ atoms (about $1.2 \AA$ ). At the same time, the water oxygen begins bonding with a surface carbon atom (inset of Fig. 5). This process results in a lower barrier than that present in the nitrogen case, which is less electronegative and forms weaker $\mathrm{H}$-bonds. Therefore, for nitrogen, dissociation of the $\mathrm{O}-\mathrm{H}$ bond in the water molecule occurs before the hydroxyl is subsequently available to bond in the surface, resulting in a larger barrier. 
The processes for water dissociation for the dopants in the present study once again differ from that of the Si case [43]. When silicon was present, it was found that not only were the final energies for dissociative adsorption more stable as opposed to the ones studied here by $\sim 2 \mathrm{eV}$ (see Table 1), but the barrier for water dissociation $(0.24 \mathrm{eV}$ ) was lowered to half of that seen for th e undoped case $(0.48 \mathrm{eV})$. This was the crucial step in subsequently observing an increase of surface hydroxylation in sliding-wall AIMD simulations [8], and is a mechanism that does not appear to be applicable to boron, nitrogen, or oxygen dopants.

\subsection{Effect of chemisorbed water and water fragments on surface hydrophilicity}

We have studied the role of different dopants on water molecular and dissociative adsorption pathways. We have found boron to spontaneously chemisorb water molecules, without need of dissociation to increase wettability. The adsorbed hydrogen, hydroxyl, and intact water molecules are expected to enhance the interaction with further water molecules through hydrogen bonding [43]. This is expected to be a major atomic-scale driving force for the increased hydrophilicity of boron- [25], nitrogen- [38], and oxygen-doped [20] DLC surfaces compared to undoped surfaces. Therefore, we studied the interactions of the most stable hydrogen, hydroxyl-, and water-containing BDD, NDD, and ODD structures with further water molecules.

First, either hydrogen or hydroxyl fragments were relaxed on atoms in the outer layer. As expected from the previous section, only some of the adsorbed configurations were stable; namely, the ones involving hydrogen atoms bonded on carbon, boron or nitrogen atoms, and the hydroxyls bonded to carbon or boron. Although $\mathrm{B}-\mathrm{H}$ and $\mathrm{N}-\mathrm{H}$ bonds are not among the most favourable configurations shown in Table 1, they are included in this section for further investigation. Once these configurations were relaxed, a water molecule was placed on top of them on several geometries to calculate the interaction with the fragments using PBE. Similarly to previous steps, the most stable configurations were then relaxed with DFT-D2 and DFT-D3. The corresponding adsorption geometries, energies and relevant partial charges are presented in Fig. 6 a-e. 
a)

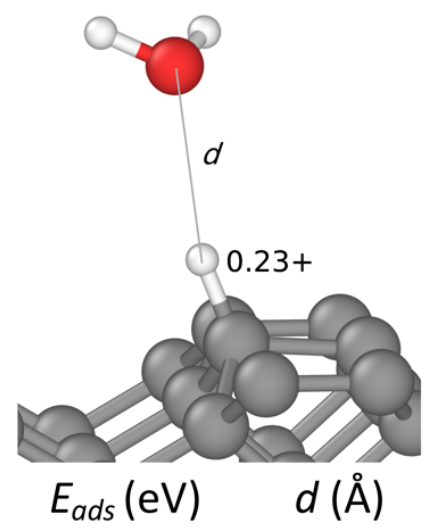

b)

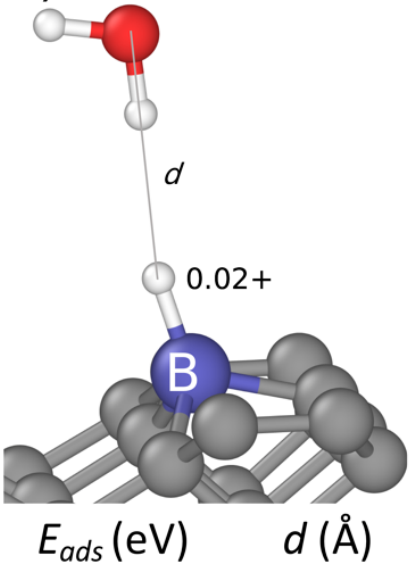

c)

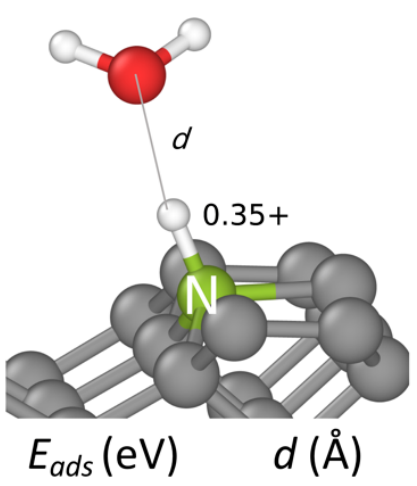

PBE:
$-0.10$

2.4

$-0.06$

3.0

$-0.37$

1.7

DFT-D2:

$-0.17$

2.3

$-0.11$

3.0

$-0.49$

1.7

$-0.10$

3.0

$-0.47$

1.7

d)

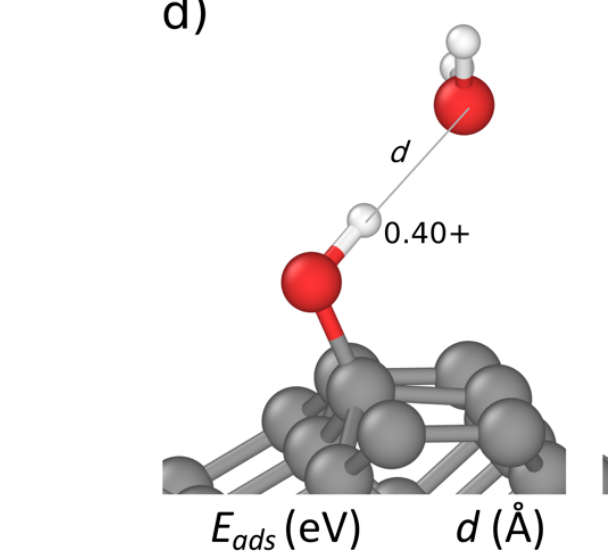

e)

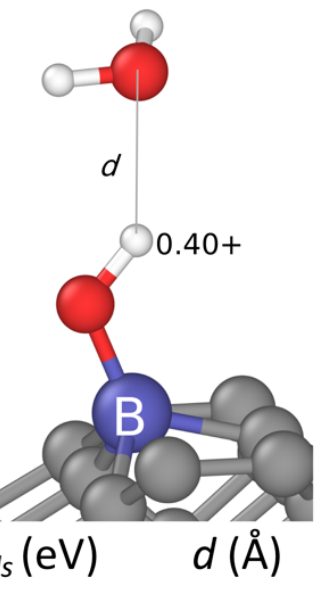

f)

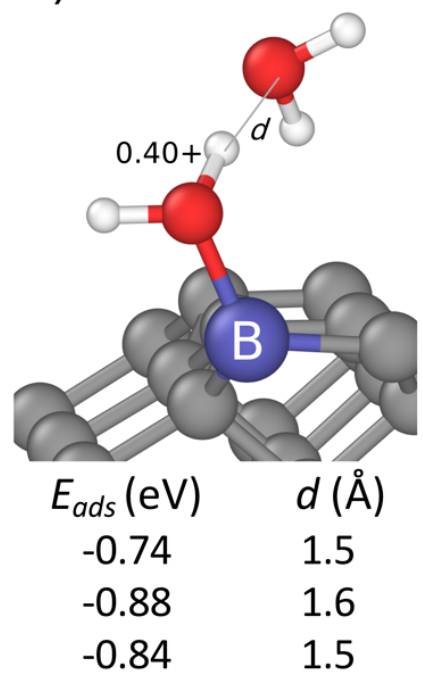

Figure 6. Relaxed configurations for water adsorption on adsorbed hydrogen (a-c), hydroxyl (d-e) and chemisorbed water (f). Only the stable systems are shown (e.g., a hydroxyl fragment is not stable adsorbed on an $O$ dopant). Distances between the water oxygen and the hydrogen in the adsorbed fragment are shown (in angstrom) for the PBE geometries, as well as the Löwdin charges of the latter. $B$ is shown in blue, $\mathrm{N}$ in green, $\mathrm{O}$ in red, $\mathrm{H}$ in white and $\mathrm{C}$ in grey.

As in the case of water adsorption on clean surfaces, it can be observed that, while the PBE results underestimate the adsorption energies compared to the vdW- 
corrected ones, they qualitatively agree in sorting the strength of the different interactions.

In general, the systems in which the surface $\mathrm{H}$ atom is more positively charged interact more strongly with the water molecules. This observation is expected due the primarily electrostatic nature of the hydrogen bonds. The system with the weakest H-bonding is the one with the hydrogen adsorbed on the B dopant (Fig. 6b). B has a similar electronegativity to $\mathrm{H}[69]$, meaning that the $\mathrm{B}-\mathrm{H}$ bond is nonpolar, and its interactions with water are very weak (DFT-D3: $-0.10 \mathrm{eV}$ ). It is the only system where the water molecule is relaxed with the $\mathrm{O}$ atom in the water molecule facing away from the surface $\mathrm{H}$ atom. Table 1 shows that hydroxylation (a1-3) is much more energetically favourable compared to hydrogenation (a4-6), meaning that when the surface contacts water it is likely to become more hydrophilic (rather than hydrophobic). For the hydrogen-terminated DLC surface (Fig. 6a), since $\mathrm{C}$ and $\mathrm{H}$ have more different electronegativities, the interaction is slightly stronger (DFT-D3: $-0.16 \mathrm{eV}$ ) than for BDD. These observations suggest that B-dopants are only likely to increase hydrophilicity (as has been observed experimentally [25]) in hydrogen-free carbon surfaces.

Only the hydrogen atom adsorbed on nitrogen (Fig. 6c) interacts more strongly with water molecules (DFT-D3: $-0.47 \mathrm{eV}$ ) than its undoped counterpart. In this system, the surface dimer is broken as the $\mathrm{N}$ outer shell is saturated with three bonds when adding the hydrogen. It presents the highest interaction energy of all hydroxylated or hydrogenated systems, interestingly even higher than water-hydroxyl interaction (Fig. 6d, DFT-D3: $-0.37 \mathrm{eV}$ ) on a clean surface. While hydroxylation is often linked with enhanced hydrophilicity $[8,43,46]$, we see the high difference in electronegativities between nitrogen and hydrogen induces a polarity that makes the latter participate in hydrogen bonding. Indeed, the experimental decrease in contact angle of N-DLC has been linked to the presence of $\mathrm{N}-\mathrm{H}$ bonds [38] (and the $\mathrm{C}-\mathrm{N}$ bonds mentioned in previous sections).

We also studied the interaction of the boron-chemisorbed water molecule on further water molecules (Fig. 6f). It is found to pull the molecule closer and draw a stronger interaction (DFT-D3: $-0.84 \mathrm{eV}$ ) than the dissociated fragments (Fig. 6e, $-0.19 \mathrm{eV}$ ), to an extent that the interaction can be considered a strong hydrogen bond [79]. This 
further supports the prospect of boron providing a mechanism for increasing hydrophilicity without an energy barrier through a two-step process: first, there is a large energy gain for spontaneous water chemisorption; then there is a strong hydrogen bond interaction between said molecule and a water interface. Together, both mechanisms result in an energy benefit of approximately $2 \mathrm{eV}$.

\section{DISCUSSION}

Direct interaction between dopants and intact water molecules (see Fig. 3) results in increased adsorption for all of the dopants compared to undoped DLC in the following order; NDD ODD < BDD according to vdW-corrected calculations, although PBE calculations indicate NDD < ODD. The higher electronegativity of $\mathrm{N}$ compared to $\mathrm{C}$ results in a polar bond with a negative partial charge on the $\mathrm{N}$ atom that attracts the water $\mathrm{H}$ atom (DFT-D3: $-0.32 \mathrm{eV}$ ). This also occurs for $\mathrm{O}$, but the dominant interaction occurs between the water molecule and the dangling bonds of the carbon in the broken heterodimer. The adsorption energy for ODD (DFT-D3: $-0.35 \mathrm{eV}$ ) is comparable to the undoped hydroxylated surface (Fig. 6d, DFT-D3: $-0.37 \mathrm{eV}$ ). For BDD, the water $\mathrm{O}$ atom donates electrons to a dative covalent bond (DFT-D3: $-1.33 \mathrm{eV}$ ), which directly traps the molecule into the surface with no energy barrier. In all cases, the energy gain is more favourable than with clean and hydrogenated undoped surfaces (DFT-D3: $0.19 \mathrm{eV}$, Fig. 3a and $-0.16 \mathrm{eV}$ Fig. 6a, respect.). As a result, all of the doped surfaces posed an increased water interaction strength compared to their undoped counterparts, prior to any potential hydroxylation. This observation is in agreement with previous experiments showing increased hydrophilicity of boron- [25], nitrogen[38], and oxygen-doped [20] surfaces compared to undoped DLC.

Compared with Si, however, doping with $\mathrm{B}, \mathrm{N}$ and $\mathrm{O}$ does not result in a significantly higher energy gain for dissociative adsorption. The presence of $\mathrm{Si}$ in a surface heterodimer shows a unique mechanism by forcing $\mathrm{sp}^{3}$ bonding that yields favourable energy gains when saturated with hydroxyls in the silicon and hydrogens carbon of the same heterodimer. It was shown, however, that the presence of dopants modifies the dissociation energies even when not directly participating in any new bond (Fig. 4 and Table 1, a9-10). 
The possibility of $\mathrm{B}$ to adopt a tetragonal conformation and directly bond to a water molecule provides two mechanisms for increasing hydrophilicity. Firstly, the net energy gain from the spontaneous chemisorption (DFT-D3: $-1.33 \mathrm{eV}$ ) offsets the increased energy barrier $(0.69 \mathrm{eV})$ for water dissociation when compared to undoped DLC $(0.48 \mathrm{eV})$. The resulting hydroxyl-water interaction is stronger than on clean surface. However, it may be unlikely a stabilized chemisorbed water molecule will dissociate given the $\sim 40 \%$ higher dissociation barrier than in undoped DLC. Moreover, boron reduces the interaction of the directly bonded hydroxyl group with water when compared to one bonded to carbon (DFT-D3: -0.24 vs. $-0.37 \mathrm{eV}$ ). As such, the observed second mechanism is perhaps more interesting; the chemisorbed water molecule interacts very strongly with new water molecules (DFT-D3: $-0.84 \mathrm{eV}$ ) through strong hydrogen bonds [79]. This two-step process results in a total energy gain of almost $2 \mathrm{eV}$ with no energy barrier and could be a mechanism at play in the increased wettability boron-doping of DLC provides in experiments [25]. On the other hand, B-H bonds are nonpolar and, if present, are likely to decrease wettability. In the sliding-wall AIMD simulations of Si-DLC lubricated by water by Kajita and Righi [8], Si dopants reduced the energy barrier for water dissociative adsorption, and the resulting increase of surface hydroxyls participated in the hydrogen bond network, dragging along a water layer. In similar circumstances, it is expected that boron dopants drag along a water layer through intact chemisorbed water molecules. To the best of our knowledge, this water bonding mechanism has not been reported before and has important implications in the design of doped coatings for tribological and biomedical applications.

Nitrogen doping does not induce hydroxylation due to a high energy barrier (1.37 eV, Fig 5). Nonetheless, water physisorption on a nitrogen dopant is more favourable than in the undoped case (DFT-D3: - $0.32 \mathrm{eV}$ vs $-0.19 \mathrm{eV}$; Fig. 3). Similarly, nitrogen-bonded hydrogens provide a higher energy gain at a water interface than any other fragment observed in this study (DFT-D3: $-0.47 \mathrm{eV}$, Fig. 6c). These two interactions could tentatively explain the higher experimental hydrophilicity of N-DLC compared to undoped DLC [38]. Indeed, the decrease in WCA in with increasing content was linked to the formation of polar $\mathrm{C}-\mathrm{N}$ and $\mathrm{N}-\mathrm{H}$ bonds [38]. 
Oxygen dopants in bridge positions yield the highest adsorption energies of clean surfaces (non-hydrogenated/hydroxylated) in this study due to surface disruption and induced dangling bonds. Despite favouring the same dissociative adsorption configuration on the surface as nitrogen dopants, they present a mechanism that results in a similar energy barrier to undoped DLC $(0.54 \mathrm{eV})$ by participating in a O$\mathrm{H} \cdots \mathrm{O}$ system with the water molecule, which facilitates the $\mathrm{O}-\mathrm{H}$ cleavage. The net energy barrier of the process (that is, taking as a starting point the energy of a noninteracting water molecule) is lower than for undoped DLC.

\section{CONCLUSIONS}

In this study, the effect of $\mathrm{B}, \mathrm{N}$ and $\mathrm{O}$ dopants on the hydrophilicity mechanisms of a (100) $2 \times 1$ reconstructed diamond surface were investigated with DFT, both for a simple PBE functional and DFT-D2 and DFT-D3 vdW corrections. For all of the dopants, the preferred substitutional positions were found to be in the surface heterodimers. The effects of the dopants on the adsorption of intact water molecules, the energy barrier for dissociative adsorption, and the interaction of the dissociated fragments with additional water molecules were investigated. $\mathrm{O}$ and $\mathrm{N}$ dopants can increase the adsorption energy of water; in the latter case, especially when an $\mathrm{N}-\mathrm{H}$ bond is present. Most notably, B can adopt a tetragonal conformation and spontaneously capture a water molecule that strongly participates in an $\mathrm{H}$-bond network. Our findings have strong implications for the development and optimisation of new coating systems and demonstrate how in silico experimentation at atomistic level can be used for tuning surface properties.

\section{CRediT AUTHORSHIP CONTRIBUTION STATEMENT}

C. A. L.: Conceptualization, Methodology, Visualization, Writing - Original Draft; J. P. E.: Conceptualization, Supervision, Writing - Review \& Editing; D. D.: Supervision, Writing Review \& Editing; M. C. R.: Conceptualization, Writing - Review \& Editing.

\section{ACKNOWLEDGEMENTS}

J.P.E. and D.D. thank the Engineering and Physical Sciences Research Council (EPSRC) for Grant EP/P030211/1 and Established Career Fellowship EP/N025954/1. C.A.L. thanks the EPSRC and Afton Chemical Corporation for Ph.D. funding via the Theory and Simulation of 
Materials-Centre for Doctoral Training (TSM-CDT) EP/L015579/1. The authors acknowledge the use of Imperial College London Research Computing Service (RCS). M.C.R. thanks HPCEUROPA3 (INFRAIA-2016-1-730897). All data reported in the paper can be obtained by emailing the corresponding author or tribology@imperial.ac.uk.

\section{REFERENCES}

[1] R. Hauert, A review of modified DLC coatings for biological applications, Diam. Relat. Mater. 12 (2003) 583-589. https://doi.org/10.1016/S09259635(03)00081-5.

[2] C.A. Love, R.B. Cook, T.J. Harvey, P.A. Dearnley, R.J.K. Wood, Diamond like carbon coatings for potential application in biological implants - A review, Tribol. Int. 63 (2013) 141-150. https://doi.org/10.1016/j.triboint.2012.09.006.

[3] A. Erdemir, C. Donnet, Tribology of diamond-like carbon films: Recent progress and future prospects, J. Phys. D. Appl. Phys. 39 (2006). https://doi.org/10.1088/0022-3727/39/18/R01.

[4] A. Erdemir, J.M. Martin, Superior wear resistance of diamond and DLC coatings, Curr. Opin. Solid State Mater. Sci. $22 \quad$ (2018) 243-254. https://doi.org/10.1016/j.cossms.2018.11.003.

[5] Z. Chen, X. He, C. Xiao, S. Kim, Effect of Humidity on Friction and Wear-A $\begin{array}{lllll}\text { Critical } & \text { Review, } & \text { Lubricants. } & 6 & \text { (2018) }\end{array}$ https://doi.org/10.3390/lubricants6030074.

[6] J. Wang, K. Zhang, F. Wang, W. Zheng, Improving frictional properties of DLC films by surface energy manipulation, RSC Adv. 8 (2018) 11388-11394. https://doi.org/10.1039/c8ra00580j.

[7] X. Wu, T. Ohana, A. Tanaka, T. Kubo, H. Nanao, I. Minami, S. Mori, Tribochemical investigation of DLC coating in water using stable isotopic tracers, Appl. Surf. Sci. $254 \quad$ (2008) 3397-3402. https://doi.org/10.1016/j.apsusc.2007.11.024.

[8] S. Kajita, M.C. Righi, A fundamental mechanism for carbon-film lubricity identified by means of ab initio molecular dynamics, Carbon N. Y. 103 (2016) 193-199. https://doi.org/10.1016/j.carbon.2016.02.078.

[9] M.-I. De, B. Bouchet, G. Zilibotti, C. Matta, M.C. Righi, L. Vandenbulcke, B. 
Vacher, J.-M.M. Martin, M.I. De Barros Bouchet, G. Zilibotti, C. Matta, M.C. Righi, L. Vandenbulcke, B. Vacher, J.-M.M. Martin, Friction of Diamond in the Presence of Water Vapor and Hydrogen Gas. Coupling Gas-Phase Lubrication and First-Principles Studies, J. Phys. Chem. C. 116 (2012) 6966-6972. https://doi.org/10.1021/jp211322s.

[10] G. Zilibotti, S. Corni, M.C. Righi, Load-induced confinement activates diamond lubrication by water, Phys. Rev. Lett. 111 (2013). https://doi.org/10.1103/PhysRevLett.111.146101.

[11] M. Stüber, L. Niederberger, F. Danneil, H. Leiste, S. Ulrich, A. Welle, M. Marin, H. Fischer, Surface topography, surface energy and wettability of magnetronsputtered amorphous carbon (a-C) films and their relevance for platelet adhesion, Adv. Eng. Mater. 9 (2007) 1114-1122. https://doi.org/10.1002/adem.200700224.

[12] M. Jelínek, K. Smetana, T. Kocourek, B. Dvořánková, J. Zemek, J. Remsa, T. Luxbacher, Biocompatibility and sp3/sp2 ratio of laser created DLC films, Mater. Sci. Eng. B Solid-State Mater. Adv. Technol. 169 (2010) 89-93. https://doi.org/10.1016/j.mseb.2010.01.010.

[13] J. Shi, Z. Gong, Y. Wang, K. Gao, J. Zhang, Friction and wear of hydrogenated and hydrogen-free diamond-like carbon films: Relative humidity dependent character, Appl. Surf. Sci. $422 \quad$ (2017) 147-154. https://doi.org/10.1016/j.apsusc.2017.05.210.

[14] C. Wei, W.J. Pan, M.S. Hung, The effects of substrate roughness and associated surface properties on the biocompatibility of diamond-like carbon films, Surf. Coatings Technol. $224 \quad$ (2013) 8-17. https://doi.org/10.1016/j.surfcoat.2013.02.038.

[15] C. Corbella, S. Portal-Marco, M. Rubio-Roy, E. Bertran, G. Oncins, M.A. Vallvé, J. Ignés-Mullol, J.L. Andjuar, Modifying surface properties of diamond-like carbon films via nanotexturing, J. Phys. D. Appl. Phys. 44 (2011) 395301. https://doi.org/10.1088/0022-3727/44/39/395301.

[16] J.Q. Liu, L.J. Li, B. Wei, F. Wen, H.T. Cao, Y.T. Pei, Effect of sputtering pressure on the surface topography, structure, wettability and tribological performance of DLC films coated on rubber by magnetron sputtering, Surf. Coatings Technol. 365 (2019) 33-40. https://doi.org/10.1016/j.surfcoat.2018.05.012.

[17] M. Grischke, A. Hieke, F. Morgenweck, H. Dimigen, Variation of the wettability 
of DLC-coatings by network modification using silicon and oxygen, Diam. Relat. Mater. 7 (1998) 454-458. https://doi.org/10.1016/s0925-9635(97)00237-9.

[18] S.C.H. Kwok, J. Wang, P.K. Chu, Surface energy, wettability, and blood compatibility phosphorus doped diamond-like carbon films, Diam. Relat. Mater. 14 (2005) 78-85. https://doi.org/10.1016/j.diamond.2004.07.019.

[19] S. Akaike, D. Kobayashi, Y. Aono, M. Hiratsuka, A. Hirata, T. Hayakawa, Y. Nakamura, Relationship between static friction and surface wettability of orthodontic brackets coated with diamond-like carbon (DLC), fluorine- or silicone-doped DLC coatings, Diam. Relat. Mater. 61 (2016) 109-114. https://doi.org/10.1016/j.diamond.2015.11.016.

[20] P. Safaie, A. Eshaghi, S.R. Bakhshi, Oxygen doping effect on the wettability of diamond-like carbon thin films, J. Non. Cryst. Solids. 471 (2017) 410-414. https://doi.org/10.1016/j.jnoncrysol.2017.06.034.

[21] C. Donnet, Recent progress on the tribology of doped diamond-like and carbon alloy coatings: A review, Surf. Coatings Technol. 100-101 (1998) 180-186. https://doi.org/10.1016/S0257-8972(97)00611-7.

[22] A. Sen, S. Barizuddin, M. Hossain, L. Polo-Parada, K.D. Gillis, S. Gangopadhyay, Preferential cell attachment to nitrogen-doped diamond-like carbon (DLC:N) for the measurement of quantal exocytosis, Biomaterials. 30 (2009) 1604-1612. https://doi.org/10.1016/j.biomaterials.2008.11.039.

[23] P.W. May, P.A. Nistor, P.W. May, Diamond thin films: giving biomedical applications a new shine, J. R. Soc. Interface. 14 (2017) 20170382. https://doi.org/10.1098/rsif.2017.0382.

[24] C. Ronning, U. Griesmeier, M. Gross, H.C. Hofsäss, R.G. Downing, G.P. Lamaze, Conduction processes in boron- and nitrogen-doped diamond-like carbon films prepared by mass-separated ion beam deposition, Diam. Relat. Mater. 4 (1995) 666-672. https://doi.org/10.1016/0925-9635(94)05219-0.

[25] S. Liza, J. Hieda, H. Akasaka, N. Ohtake, Y. Tsutsumi, A. Nagai, T. Hanawa, Deposition of boron doped DLC films on TiNb and characterization of their mechanical properties and blood compatibility, 18 (2017) 76-87. https://doi.org/10.1080/14686996.2016.1262196.

[26] J.G. Buijnsters, M. Tsigkourakos, T. Hantschel, F.O.V. Gomes, T. Nuytten, P. Favia, H. Bender, K. Arstila, J.P. Celis, W. Vandervorst, Effect of Boron Doping on the Wear Behavior of the Growth and Nucleation Surfaces of Micro- and 
Nanocrystalline Diamond Films, ACS Appl. Mater. Interfaces. 8 (2016) 2638126391. https://doi.org/10.1021/acsami.6b08083.

[27] R. Prioli, S.I. Zanette, A.O. Caride, D.F. Franceschini, F.L. Freire, Atomic force microscopy of amorphous hydrogenated carbon-nitrogen films deposited by radio-frequency-plasma decomposition of methane-ammonia gas mixtures, Cit. J. Vac. Sci. Technol. A. 14 (1996) 2351. https://doi.org/10.1116/1.580021.

[28] F. Wen, N. Huang, Y.X. Leng, J. Wang, H. Sun, Y.J. Li, Z.W. Wang, Studies of the composition, mechanical and electrical properties of $\mathrm{N}$-doped carbon films prepared by DC-MFCAD, in: Nucl. Instruments Methods Phys. Res. Sect. B Beam Interact. with Mater. Atoms, 2006: pp. 324-327. https://doi.org/10.1016/j.nimb.2005.08.070.

[29] X. Yan, T. Xu, G. Chen, S. Yang, H. Liu, Study of structure, tribological properties and growth mechanism of DLC and nitrogen-doped DLC films deposited by electrochemical technique, Appl. Surf. Sci. 236 (2004) 328-335. https://doi.org/10.1016/j.apsusc.2004.05.005.

[30] N. Nakamura, T. Itani, H. Chiba, K. Watanabe, K. Kurihara, Effects of O2 gas addition on diamond-like carbon film deposition, Mater. Res. Soc. Symp. - Proc. 593 (2000) 415-419. https://doi.org/10.1557/proc-593-415.

[31] P. Safaie, A. Eshaghi, S.R. Bakhshi, Structure and mechanical properties of oxygen doped diamond-like carbon thin films, Diam. Relat. Mater. 70 (2016) $91-$ 97. https://doi.org/10.1016/j.diamond.2016.10.008.

[32] D. Neerinck, P. Persoone, M. Sercu, A. Goel, D. Kester, D. Bray, Diamond-like nanocomposite coatings (a-C:H/a-Si:O) for tribological applications, Diam. Relat. Mater. 7 (1998) 468-471. https://doi.org/10.1016/s0925-9635(97)00201$\mathrm{x}$.

[33] M. Evaristo, R. Azevedo, C. Palacio, A. Cavaleiro, Influence of the silicon and oxygen content on the properties of non-hydrogenated amorphous carbon coatings, Diam. Relat. Mater. 70 (2016) 201-210. https://doi.org/10.1016/j.diamond.2016.10.024.

[34] M. Kalin, M. Polajnar, The wetting of steel, DLC coatings, ceramics and polymers with oils and water: The importance and correlations of surface energy, surface tension, contact angle and spreading, Appl. Surf. Sci. 293 (2014) 97-108. https://doi.org/10.1016/j.apsusc.2013.12.109.

[35] G.Q. Yu, B.K. Tay, Z. Sun, Fluorinated amorphous diamond-like carbon films 
deposited by plasma-enhanced chemical vapor deposition, Surf. Coatings Technol. 191 (2005) 236-241. https://doi.org/10.1016/j.surfcoat.2004.04.060.

[36] P. Yang, N. Huang, Y.X. Leng, Z.Q. Yao, H.F. Zhou, M. Maitz, Y. Leng, P.K. Chu, Wettability and biocompatibility of nitrogen-doped hydrogenated amorphous carbon films: Effect of nitrogen, Nucl. Instruments Methods Phys. Res. Sect. B Beam Interact. with Mater. Atoms. 242 (2006) 22-25. https://doi.org/10.1016/j.nimb.2005.08.081.

[37] Definitions for Hydrophilicity, Hydrophobicity, and Superhydrophobicity: Getting the Basics Right, (2014). https://doi.org/10.1021/jz402762h.

[38] M.H. Ahmed, J.A. Byrne, Effect of surface structure and wettability of DLC and N-DLC thin films on adsorption of glycine, Appl. Surf. Sci. 258 (2012) 51665174. https://doi.org/10.1016/j.apsusc.2012.01.162.

[39] J. Andersson, R.A. Erck, A. Erdemir, Frictional behavior of diamondlike carbon films in vacuum and under varying water vapor pressure, Surf. Coatings Technol. 163-164 (2003) 535-540. https://doi.org/10.1016/S02578972(02)00617-5.

[40] J. Wang, L. Shang, X. Li, Z. Lu, G. Zhang, Quantifying Macroscopic Friction of Diamond-like Carbon Films by Microscopic Adsorption and Removal of Water Molecules, (2017). https://doi.org/10.1021/acs.langmuir.7b02613.

[41] O. Manelli, S. Corni, M.C. Righi, Water Adsorption on Native and Hydrogenated Diamond (001) Surfaces, J. Phys. Chem. C. 114 (2010) 7045-7053. https://doi.org/10.1021/jp910971e.

[42] G. Levita, S. Kajita, M.C. Righi, Water adsorption on diamond (111) surfaces: an ab initio study, Carbon N. Y. 127 (2018) 533-540. https://doi.org/10.1016/j.carbon.2017.11.010.

[43] S. Kajita, M.C. Righi, Insigths into the Tribochemistry of Silicon-doped CarbonBased Films by Ab Initio Analysis of Water-Surface Interactions, Tribol. Lett. 61 (2016) 17. https://doi.org/10.1007/s11249-015-0631-1.

[44] L. Sirghi, V. Tiron, M. Dobromir, Friction at single-asperity contacts between hydrogen-free diamond-like carbon thin film surfaces, Diam. Relat. Mater. 52 (2015) 38-42. https://doi.org/10.1016/j.diamond.2014.12.007.

[45] F. Zhao, H.X. Li, L. Ji, Y.F. Mo, W.L. Quan, W. Du, H.D. Zhou, J.M. Chen, Superlow friction behavior of Si-doped hydrogenated amorphous carbon film in water environment, Surf. Coatings Technol. 203 (2009) 981-985. 
https://doi.org/10.1016/j.surfcoat.2008.09.025.

[46] Z. Futera, T. Watanabe, Y. Einaga, Y. Tateyama, First Principles Calculation Study on Surfaces and Water Interfaces of Boron-Doped Diamond, 118 (2014) 22040-22052. https://doi.org/10.1021/jp506046m.

[47] R. Jaimes, J. Vazquez-Arenas, I. González, M. Galván, Delimiting the boron influence on the adsorptive properties of water and $\mathrm{OH}$ radicals on $\mathrm{H}$-terminated Boron Doped Diamond catalysts: A Density Functional Theory analysis, Surf. Sci. 653 (2016) 27-33. https://doi.org/10.1016/j.susc.2016.04.018.

[48] S. Zhao, K. Larsson, Theoretical Study of the Energetic Stability and Geometry of Terminated and B-Doped Diamond (111) Surfaces, J. Phys. Chem. C. 118 (2014) 1944-1957. https://doi.org/10.1021/jp409278x.

[49] X. Wang, C. Wang, X. Shen, K. Larsson, F. Sun, DFT calculations of energetic stability and geometry of O-terminated B-and N-doped diamond (111)-1×1 surfaces, J. Phys. Condens. Matter. $31 \quad$ (2019) 265002. https://doi.org/10.1088/1361-648X/ab152f.

[50] G. Zilibotti, M.C. Righi, M. Ferrario, Ab initio study on the surface chemistry and nanotribological properties of passivated diamond surfaces, Phys. Rev. B. 79 (2009) 075420. https://doi.org/10.1103/PhysRevB.79.075420.

[51] P. Krüger, J. Pollmann, Dimer reconstruction of diamond, Si, and Ge (001) surfaces, Phys. Rev. Lett. $74 \quad$ (1995) 1155-1158. https://doi.org/10.1103/PhysRevLett.74.1155.

[52] J. Zemek, J. Houdkova, P. Jiricek, M. Jelinek, Surface and in-depth distribution of sp2 and sp3 coordinated carbon atoms in diamond-like carbon films modified by argon ion beam bombardment during growth, Carbon. 134 (2018) 71-79. https://doi.org/10.1016/j.carbon.2018.03.072.

[53] R. Samlenski, C. Haug, R. Brenn, C. Wild, R. Locher, P. Koidl, Characterisation and lattice location of nitrogen and boron in homoepitaxial CVD diamond, Diam. Relat. Mater. 5 (1996) 947-951. https://doi.org/10.1016/0925-9635(95)00471-8.

[54] J. Robertson, C.A. Davis, Nitrogen doping of tetrahedral amorphous carbon, Diam. Relat. Mater. 4 (1995) 441-444. https://doi.org/10.1016/09259635(94)05265-4.

[55] Sholihun, H.P. Kadarisman, P. Nurwantoro, Density-functional-theory calculations of formation energy of the nitrogen-doped diamond, Indones. J. Chem. 18 (2018) 749-754. https://doi.org/10.22146/ijc.26785. 
[56] P. Giannozzi, S. Baroni, N. Bonini, M. Calandra, R. Car, C. Cavazzoni, D. Ceresoli, G.L. Chiarotti, M. Cococcioni, I. Dabo, A. Dal Corso, S. De Gironcoli, S. Fabris, G. Fratesi, R. Gebauer, U. Gerstmann, C. Gougoussis, A. Kokalj, M. Lazzeri, L. Martin-Samos, N. Marzari, F. Mauri, R. Mazzarello, S. Paolini, A. Pasquarello, L. Paulatto, C. Sbraccia, S. Scandolo, G. Sclauzero, A.P. Seitsonen, A. Smogunov, P. Umari, R.M. Wentzcovitch, QUANTUM ESPRESSO: A modular and open-source software project for quantum simulations of materials, J. Phys. Condens. Matter. 21 (2009). https://doi.org/10.1088/0953-8984/21/39/395502.

[57] P. Giannozzi, O. Andreussi, T. Brumme, O. Bunau, M. Buongiorno, M. Nardelli, R. Calandra, C. Car, D. Cavazzoni, M. Ceresoli, M. Cococcioni, N. Colonna, I. Carnimeo, A. Dal Corso, S. de Gironcoli, R. P Delugas, A. DiStasio, A. Ferretti, A. Floris, G. Fratesi, G. Fugallo, R. Gebauer, U. Gerstmann, F. Giustino, T. Gorni, J. Jia, M. Kawamura, H.-Y. Ko, A. Kokalj, E. Küçükbenli, M. Lazzeri, M. Marsili, N. Marzari, F. Mauri, N.L. Nguyen, H.-V. Nguyen, A. Otero-de-la-Roza, L. Paulatto, S. Poncé, D. Rocca, R. Sabatini, B. Santra, M. Schlipf, A.P. Seitsonen, A. Smogunov, I. Timrov, T. Thonhauser, P. Umari, N. Vast, X. Wu, S. Baroni, Advanced capabilities for materials modelling with Quantum ESPRESSO, J. Phys. Condens. Matter. $29 \quad$ (2017) 465901. https://doi.org/10.1088/1361-648X/aa8f79.

[58] J.P. Perdew, K. Burke, M. Ernzerhof, Generalized gradient approximation made simple, Phys. Rev. Lett. $77 \quad$ (1996) 3865-3868. https://doi.org/10.1103/PhysRevLett.77.3865.

[59] W. Kohn, L.J. Sham, Self-Consistent Equations Including Exchange and Correlation Effects, Phys. Rev. 140 (1965) 1133-1138.

[60] J.P. Perdew, Y. Wang, Accurate and simple analytic representation of the electron-gas correlation energy, Phys. Rev. B. 45 (1992) 13244-13249. https://doi.org/10.1103/PhysRevB.45.13244.

[61] S. Grimme, Semiempirical GGA-type density functional constructed with a longrange dispersion correction, J. Comput. Chem. 27 (2006) 1787-1799. https://doi.org/10.1002/jcc.20495.

[62] V. Barone, M. Casarin, D. Forrer, M. Pavone, M. Sambi, A. Vittadini, Role and effective treatment of dispersive forces in materials: Polyethylene and graphite crystals as test cases, J. Comput. Chem. 30 (2009) 934-939. 
https://doi.org/10.1002/jcc.21112.

[63] S. Grimme, J. Antony, S. Ehrlich, H. Krieg, A consistent and accurate ab initio parametrization of density functional dispersion correction (DFT-D) for the 94 elements $\mathrm{H}-\mathrm{Pu}, \quad J . \quad$ Chem. Phys. $132 \quad$ (2010) 154104. https://doi.org/10.1063/1.3382344.

[64] D. Vanderbilt, Soft self-consistent pseudopotentials in a generalized eigenvalue formalism, Phys. Rev. B. 41 (1990) 7892-7895. https://doi.org/10.1103/PhysRevB.41.7892.

[65] H.J. Monkhorst, J.D. Pack, Special points for Brillouin-zone integrations, Phys. Rev. B. 13 (1976) 5188-5192. https://doi.org/10.1103/PhysRevB.13.5188.

[66] A. Stukowski, Visualization and analysis of atomistic simulation data with OVITO-the Open Visualization Tool, Model. Simul. Mater. Sci. Eng. 18 (2010) 015012. https://doi.org/10.1088/0965-0393/18/1/015012.

[67] L. Mayrhofer, G. Moras, N. Mulakaluri, S. Rajagopalan, P.A. Stevens, M. Moseler, Fluorine-Terminated Diamond Surfaces as Dense Dipole Lattices: The Electrostatic Origin of Polar Hydrophobicity, J. Am. Chem. Soc. 138 (2016) 4018-4028. https://doi.org/10.1021/jacs.5b04073.

[68] F. Birch, Finite elastic strain of cubic crystals, Phys. Rev. 71 (1947) 809-824. https://doi.org/10.1103/PhysRev.71.809.

[69] J. Emsley, The elements, Clarendon Press, 1998.

[70] K. Czelej, M.R. Zemła, P. Kamińska, P. Śpiewak, K.J. Kurzydłowski, Clustering of hydrogen, phosphorus, and vacancies in diamond: A density functional theory analysis, Phys. Rev. B. $98 \quad$ (2018) 075208. https://doi.org/10.1103/PhysRevB.98.075208.

[71] M. De La Pierre, M. Bruno, C. Manfredotti, F. Nestola, M. Prencipe, C. Manfredotti, The (100), (111) and (110) Surfaces of Diamond: an Ab Initio B3LYP Study, Mol. Phys. $112 \quad$ (2014) 1030-1039. https://doi.org/10.1080/00268976.2013.829250.

[72] R. Tran, Z. Xu, B. Radhakrishnan, D. Winston, W. Sun, K.A. Persson, S.P. Ong, Data Descriptor: Surface energies of elemental crystals, Sci. Data. 3 (2016). https://doi.org/10.1038/sdata.2016.80.

[73] G. Henkelman, H. Jónsson, Improved tangent estimate in the nudged elastic band method for finding minimum energy paths and saddle points, J. Chem. Phys. 113 (2000) 9978. https://doi.org/10.1063/1.1323224. 
[74] G. Henkelman, B.P. Uberuaga, H. Jónsson, A climbing image nudged elastic band method for finding saddle points and minimum energy paths, J. Chem. Phys. 113 (2000) 9901. https://doi.org/10.1063/1.1329672.

[75] P.O. Löwdin, On the non-orthogonality problem connected with the use of atomic wave functions in the theory of molecules and crystals, J. Chem. Phys. 18 (1950) 365-375. https://doi.org/10.1063/1.1747632.

[76] O. Sharifahmadian, F. Mahboubi, A comparative study of microstructural and tribological properties of N-DLC/DLC double layer and single layer coatings deposited by DC-pulsed PACVD process, Ceram. Int. 45 (2019) 7736-7742. https://doi.org/10.1016/j.ceramint.2019.01.076.

[77] B. Cordero, V. Gómez, A.E. Platero-Prats, M. Revés, J. Echeverría, E. Cremades, F. Barragán, S. Alvarez, Covalent radii revisited, J. Chem. Soc. Dalt. Trans. (2008) 2832-2838. https://doi.org/10.1039/b801115j.

[78] J.O. Hansen, R.G. Copperthwaite, T.E. Derry, J.M. Pratt, A tensiometric study of diamond (111) and (110) faces, J. Colloid Interface Sci. 130 (1989) 347-358. https://doi.org/10.1016/0021-9797(89)90114-8.

[79] S.J. Grabowski, Theoretical studies of strong hydrogen bonds, Annu. Reports Prog. Chem. - Sect. C. 102 (2006) 131-165. https://doi.org/10.1039/b417200k. 